\title{
Cerebral toxoplasmosis in a diffuse large B cell lymphoma patient
}

\author{
Lina Savsek ${ }^{1}$, Tanja Ros Opaskar² \\ ${ }^{1}$ Department of Neurology, General Hospital Celje, Celje, Slovenia \\ ${ }^{2}$ Unit of Neurology, Institute of Oncology Ljubljana, Ljubljana, Slovenia \\ Radiol Oncol 2016; 50(1): 87-93.
}

Received 27 May 2014

Accepted 21 August 2014

Correspondence to: Tanja Ros Opaskar, M.D., M.Sc., Unit of Neurology, Institute of Oncology Ljubljana, Ljubljana, Slovenia. E-mail: tros@onko-i.si

Disclosure: No potential conflicts of interest were disclosed.

Background. Toxoplasmosis is an opportunistic protozoal infection that has, until now, probably been an underestimated cause of encephalitis in patients with hematological malignancies, independent of stem cell or bone marrow transplant. $T$ and $B$ cell depleting regimens are probably an important risk factor for reactivation of a latent toxoplasma infection in these patients.

Case report. We describe a 62-year-old HIV-negative right-handed Caucasian female with systemic diffuse large B cell lymphoma who presented with sudden onset of high fever, headache, altered mental status, ataxia and findings of pancytopenia, a few days after receiving her final, $8^{\text {th }}$ cycle of rituximab, cyclophosphamide, vincristine, doxorubicin, prednisolone (R-CHOP) chemotherapy regimen. A progression of lymphoma to the central nervous system was suspected. MRI of the head revealed multiple on T2 and fluid attenuated inversion recovery (FLAIR) hyperintense parenchymal lesions with mild surrounding edema, located in both cerebral and cerebellar hemispheres that demonstrated moderate gadolinium enhancement. The polymerase chain reaction on cerebrospinal fluid (CSF PCR) was positive for Toxoplasma gondii. The patient was diagnosed with toxoplasmic encephalitis and successfully treated with sulfadiazine, pyrimethamine and folic acid. Due to the need for maintenance therapy with rituximab for lymphoma remission, the patient now continues with secondary prophylaxis of toxoplasmosis.

Conclusions. With this case report, we wish to emphasize the need to consider cerebral toxoplasmosis in patients with hematological malignancies on immunosuppressive therapy when presenting with new neurologic deficits. In such patients, there are numerous differential diagnoses for cerebral toxoplasmosis, and the CNS lymphoma is the most difficult among all to distinguish it from. If left untreated, cerebral toxoplasmosis has a high mortality rate; therefore early recognition and treatment are of essential importance.

Key words: toxoplasmosis;,cerebral; lymphoma, B-cell; rituximab; hosts, immunocompromised; magnetic resonance imaging; treatment

\section{Introduction}

Toxoplasmosis is caused by an infection with the obligate intracellular parasite Toxoplasma gondii. The general assumption is that approximately 25 to $30 \%$ of the world's human population is infected by Toxoplasma ${ }^{1}$, which makes it one of the most common human infections in the world. The prevalences vary widely between countries, with the lowest seroprevalences observed in the coun- tries of North America, South East Asia, Northern Europe and the Sahelian countries of Africa (between $10-30 \%$ ). Toxoplasmosis is highly prevalent in Latin America and tropical African countries, while in the countries of Central and Southern Europe, including Slovenia, moderate seroprevalences have been found (30-50\%). ${ }^{1}$

Toxoplasma gondii has a complex lifecycle involving felines, in which the sexual phase is completed, as it's definite host. Oocysts shed in feline 

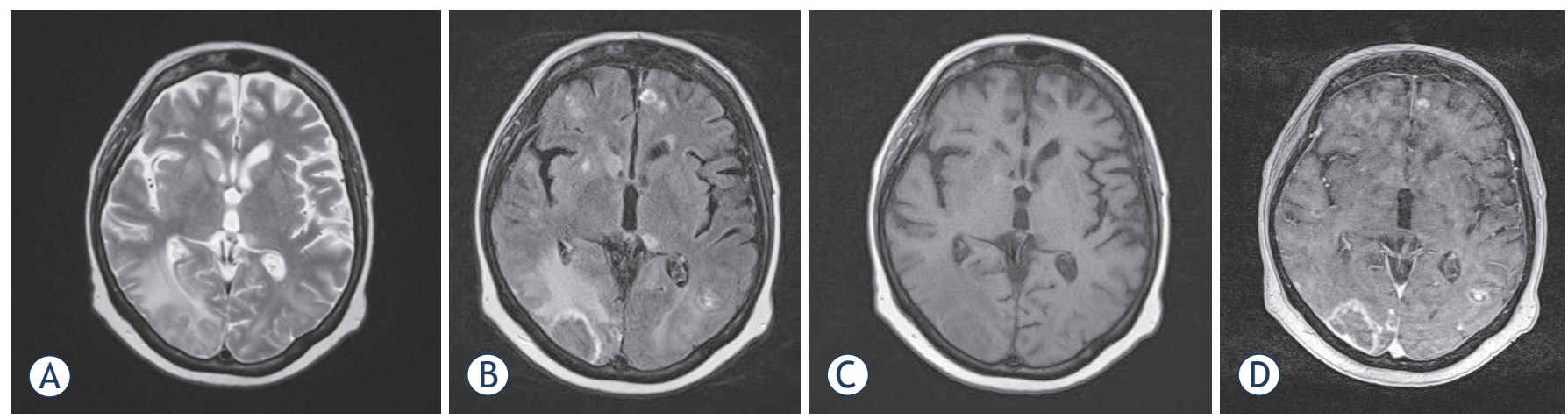

FIGURE 1. (A) MRI at time of diagnosis demonstrating multiple T2 and (B) fluid attenuated inversion recovery (FLAIR) hyperintense parenchymal lesions, located in both cerebral and cerebellar hemispheres, with mild surrounding edema. (C) On Tl sequences, these lesions were hypointense. (D) After contrast administration, only moderate rim enhancement was seen.

feces can infect a wide range of animals, including birds, rodents, grazing domestic animals, and humans. ${ }^{2}$ Humans usually get infected either by the ingestion of tissue cysts in infected meat or by ingestion of soil, water, or food contaminated with sporulated oocysts derived from the environment or, less frequently, directly from feline feces. ${ }^{1}$ In addition to oral transmission, direct transmission of the parasite by blood or organ products during transplantation takes place at a low rate. Apart from horizontal transmission, a vertical route of transmission is also recognized; parasite transmission to the fetus occurs in about one-third of pregnant women with primary toxoplasmic infection. ${ }^{1.2}$

In the immunocompetent host, the infection is usually rapidly cleared and only about $10-20 \%$ of infected individuals present with a self-limited and nonspecific illness, that only rarely requires therapy ${ }^{1-3}$ In the immunocompromised host, the immune factors necessary to control the spread of the infection are lacking and the disease can be lifethreatening. In these individuals, toxoplasmosis almost always happens as a result of reactivation of a latent infection. The most common site affected by toxoplasmosis is the central nervous system (CNS). Besides CNS, multiple other organs may be involved, including the lungs, gastrointestinal tract, pancreas, skin, eyes, heart, and liver. With the advent of the human immunodeficiency virus (HIV) pandemic, toxoplasmic encephalitis has become one of the most frequent opportunistic infections. Other heavily immunocompromised patients like those after allogeneic stem cell transplantation (SCT) or previous $\mathrm{T}$ cell depleting treatment regimens (e.g. with fludarabine or alemtuzumab) are also at high risk for opportunistic infections. If left untreated, the infection with Toxoplasma gondii in such individuals is usually fatal.

\section{Case report}

A 62-year-old HIV-negative right-handed Caucasian female with systemic diffuse large B cell lymphoma (DLBCL) presented to our oncology clinic in the beginning of September 2013 with high fever, headache and altered mental status.

Her past medical history included arterial hypertension and type 2 diabetes mellitus, both well controlled with medications. Since 2010, she was treated for marginal zone B cell lymphoma stage IV A, involving the spleen, bone marrow and lymph nodes. A splenectomy was performed in July 2011. At that time, no other treatment was administered due to clinical remission. In August 2012, the disease progressed and she was started on chemotherapy with rituximab and chlorambucil (R-LP), but was switched after only two cycles of R-LP to fludarabine and cyclophosphamide in combination with rituximab (R-FC) due to further disease progression. During this time, she suffered an episode of cutaneous herpes zoster, so valacyclovir prophylaxis was initiated. In January 2013, a transformation into DLBCL was confirmed and rituximab, cyclophosphamide, vincristine, doxorubicin and prednisolone (R-CHOP) chemotherapy regimen introduced. After 4 cycles of R-CHOP, disease remission was achieved and chemotherapy continued until the beginning of August 2013, when a neutropenia was noted and prophylactic therapy with ciprofloxacin and fluconazole initiated. Only two days later she had to be hospitalized in a regional hospital because of febrile neutropenia due to urinary infection with $E$. Coli, which was successfully treated with amoxicilline/clavulanic acid. At the same time, a left posterior tibial vein thrombosis was discovered and therapy with low molecular weight heparin (LMWH) initiated. 
By the end of August 2013 she had received her $8^{\text {th }}$, final cycle of R-CHOP. Only five days after, the patient started complaining of headache with photophobia and was admitted to a regional hospital a day later with high fever, confusion and pancytopenia. A head CT scan revealed diffuse hypodensities involving the right parieto-temporo-occipital region, spreading into the frontal lobe and across the corpus callosum into the left parieto-occipital lobe. A hypodensity in the central region of the cerebellum was present as well. There were no midline brain shifts or herniations and the ventricular system was normal. Progression of the lymphoma to the CNS was suspected and the patient transferred to our oncology clinic for further evaluation and management.

At the time of our first neurological consultation, the patient was conscious but apathetic, with elements of dysexecutive syndrome. She had poor orientation in time and situation, seemed to fill in the memory gaps with confabulations and exhibited finger agnosia. Her speech was normal. A mini mental state examination (MMSE) test could not be applied at that time due to the lack of attention needed to complete the task. A left homonymous hemianopsia, denser in the lower quadrant, was clinically suspected. A slight mask-like appearance of the face was noted. The rest of the cranial nerve examination revealed no abnormalities. Meningeal signs were absent. A mild pyramidal weakness of the right upper extremity was present and she exhibited a moderate symmetrical ataxia along with dysmetria of all four extremities. The plantar responses were flexor. There were no marked sensory deficits. The clinical picture indicated bilateral multifocal cerebral cortico-subcortical involvement along with damage to the cerebellum.

She was started on antiedematous therapy with $20 \%$ mannitol solution. A diagnostic lumbar puncture was performed and the cerebrospinal fluid (CSF) analysis revealed an elevated white cell count of 27 cells ( 1 neutrophil, 20 lymphocytes, 6 monocytes), with increased lactate, LDH and protein concentration, but normal glucose level. Cytological analysis of the CSF with Giemsa staining and flow cytometric immunophenotypisation revealed no malignant cells, but there were signs of reactive pleocytosis with $\mathrm{T}$ cell predominance.

Magnetic resonance imaging (MRI) of the head revealed multiple T2 and fluid attenuated inversion recovery (FLAIR) hyperintense parenchymal lesions with mild surrounding edema, located in both cerebral and cerebellar hemispheres, with the largest lesion located in the right occipital lobe.

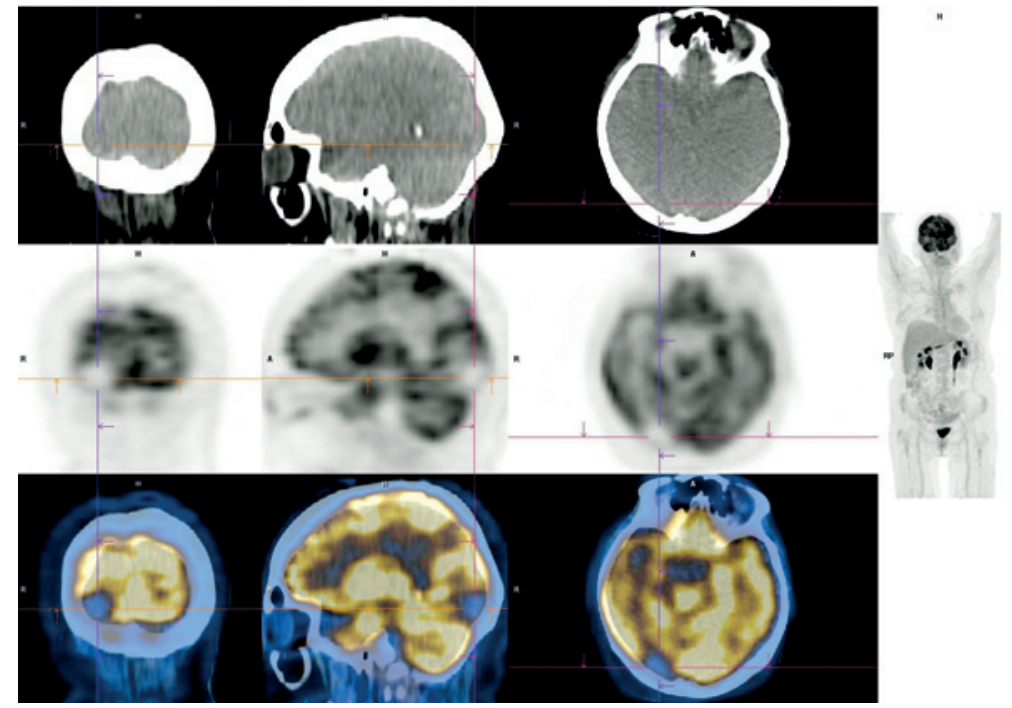

FIGURE 2. Full body 18F-FDG PET/CT revealing focal hypometabolism, corresponding to toxoplasma lesions. The largest lesion is seen in the right occipital lobe.

The lesions were hypointense on $\mathrm{T} 1$ sequences and demonstrated moderate rim enhancement after contrast administration (Figure 1).

The polymerase chain reaction on cerebrospinal fluid (CSF PCR) was positive for Toxoplasma gondii and serological analysis revealed borderline positive $\operatorname{IgG}$ and negative $\operatorname{IgM}$ antibodies. Microbiological assays for other bacteria, fungus or viruses, such as Borrelia burgdorferi, Mycobacterium tuberculosis, Cryptococcus neoformans, herpes simplex virus type 1 and 2, varicella zoster virus, enteroviruses, polioviruses, tick-borne encephalitis virus, JC virus, Epstein-Barr virus, and cytomegalovirus were negative.

There was no apparent evidence of toxoplasmosis in other organs. A full body 18F-FDG PET/CT showed remission of lymphoma and local hypometabolism in the right occipital lobe, corresponding to the location of the largest toxoplasma lesion (Figure 2).

Based on these findings, the patient was diagnosed with toxoplasmic encephalitis and therapy with sulfadiazine, pyrimethamine and folic acid was administered. Due to an underlying mild cardiomyopathy, caused by anthracycline therapy in the past, the patient suffered an acute heart failure episode in the beginning of treatment, which was managed conservatively. This condition was not directly associated with toxoplasma infection and was caused by fluid volume overload. After a few days of antibiotic therapy the patient slowly began improving. She became more alert and her cognitive status improved. A follow-up MRI of the head 

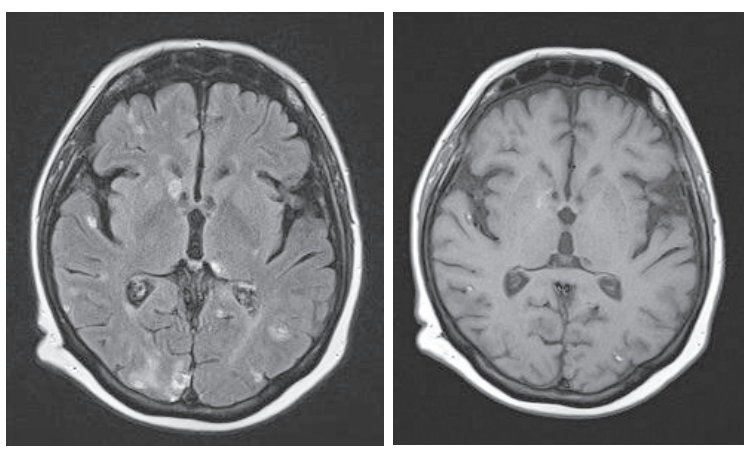

FIGURE 3. Lesion size and edema reduction after 6 weeks of intensive antibiotic therapy, as demonstrated by (A) fluid attenuated inversion recovery (FLAIR) and (B) T1 sequence.

6 weeks later showed reduction of edema and lesion size, with hemorrhagic inclusions in some of the lesions (Figure 3.) After 6 weeks of intensive antibiotic treatment we started with maintenance therapy with rituximab for lymphoma remission and continued with prophylactic doses of sulfadiazine, pyrimethamine and folic acid.

Three months after the first presentation, the patient was seen again in the neurooncology outpatient clinic for a follow-up. There were only mild cognitive deficits still present and she scored 28/30 points on MMSE. In January 2014, follow-up MRI of the head showed further reduction of brain lesions, this time without any visible hemorrhagic inclusions (Figure 4.)

Patient's written consent to publish this case report has been obtained.

\section{Discussion}

In patients with malignancies, especially those after previous allogeneic bone marrow or stem cell
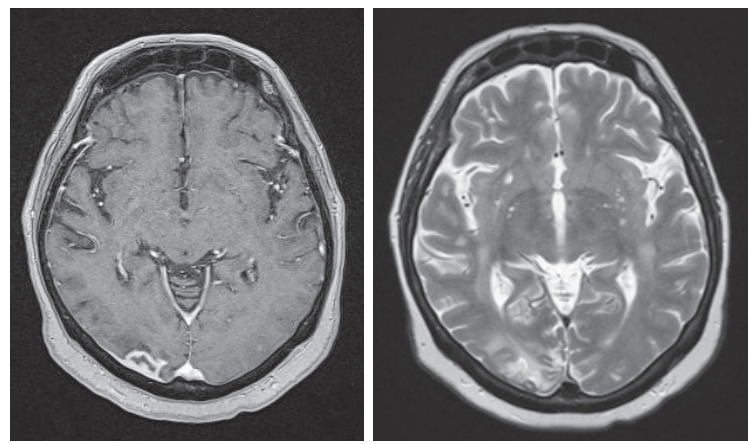

FIGURE 4. Follow-up MRI after 4 months reveals further reduction of lesion size. (A) T1 sequence + gadolinium, (B) T2 sequence. transplantation, the incidence of various CNS infections may be up to $15 \% .^{4}$ There are several risk factors for CNS infection in these individuals, depending on the underlying malignancy, its treatment and various other factors. Among causative organisms, Toxoplasma gondii is the most prevalent. ${ }^{4}$

The majority of clinical experience with cerebral toxoplasmosis was acquired in HIV/AIDS patients, in whom the risk of reactivation of latent infection with $T$. gondii is greatest when the CD4+ T-cell count decreases below 100 cells/uL. ${ }^{5}$ Cerebral toxoplasmosis is also a rare, but well known opportunistic infection in bone marrow transplant (BMT) recipients $^{6}$, as well as in solid organ transplant recipients. ${ }^{7}$

Clinical presentation of cerebral toxoplasmosis is unspecific, ranging from altered mental status, fever, seizures and headache to focal neurologic deficits, including motor deficits, cranial nerve palsies, visual field defects, aphasia, movement disorders and cerebellar dysfunction.

The gray-white matter junction, basal ganglia and thalamus are the areas predisposed to cerebral toxoplasmosis; however, the brainstem and the corpus callosum may also be involved. The lesions are usually characterized by a central zone of necrosis containing only few organisms. The central zone is surrounded by a hypervascular intermediate zone, comprised of numerous inflammatory cells mixed with tachyzoites and encysted organisms, the latter being the predominating ingredient of the final, peripheral zone. ${ }^{8}$ On nonenhanced CT scans, the lesions are usually hypo- to isodense to grey matter with surrounding vasogenic edema and mass effect, while solitary or multiple solid, nodular or ring enhancing lesions are demonstrated on contrast-enhanced CT scans. ${ }^{8,9}$ The method of choice for evaluation of mass lesions in the brain remains MRI. On MRI scans, a so-called "target sign" on T2 and FLAIR images, consisting of at least three alternating zones, is usually found. The classic constellation consists of a hypointense core surrounded by an intermediate hyperintense region and a peripheral hypointense rim, delineated by surrounding edema. Contrast T1-weighted images show an inverse appearance with ring enhancement of the inflammatory zone. ${ }^{8,9}$ A highly specific, but relatively insensitive finding for cerebral toxoplasmosis, seen in less than $30 \%$ of cases, is an asymmetric target sign, exhibiting an off-centre nodular lesion along the wall of the enhancing rim. ${ }^{8,10}$

Our patient had multiple parenchymal lesions on MRI with only moderate enhancement and surrounding edema. In patients on immunosup- 
pressant therapy, such as those after bone marrow transplant (BMT), cerebral toxoplasmosis may manifest differently than in patients with HIV/ AIDS. Due to a more global loss of immune cells, these patients are usually unable to build a sufficient immune inflammatory response at the bloodbrain barrier, therefore allowing for passage of gadolinium and vasogenic edema. However, due to a more global loss of the immune system, most of the parenchymal lesions are non-enhancing, although mass effect and edema are usually present, and there is only subtle enhancement along the meninges. ${ }^{8,11}$ In post-BMT patients, parenchymal lesions may undergo hemorrhagic transformation $^{8}$, as had our patient's lesions.

There are numerous differential diagnoses for cerebral toxoplasmosis in immunocompromised patients, among which CNS lymphoma can often be the most difficult to distinguish it from. Features suggestive of cerebral toxoplasmosis are subcortical location, multiplicity, and asymmetric target sign. ${ }^{8,10}$ In addition to standard MR imaging, MR spectroscopy, perfusion and diffusion MRI, thallium-201 SPECT or 18F-FDG PET/CT ${ }^{8,12-14}$ can be of further aid in distinguishing cerebral toxoplasmosis from lymphoma. ${ }^{8,12-14}$ On 18F-FDG/PET CT scan, which provides remarkable accuracy for detection, treatment monitoring and follow-up of patients with systemic lymphoma as well as of patients with primary central nervous system lymphoma ${ }^{15}$, the standardised uptake for cerebral lesions is much higher than in toxoplasma lesions ${ }^{8}$.

Cerebral toxoplasmosis in HIV-negative patients has been most commonly described in patients after BMT due to underlying lymphoma or leukemia. There are only rare reports of toxoplasmosis in patients with hematologic malignancies, independent of BMT. ${ }^{16,17}$ Despite clinical advancements and good safety profiles, chemotherapeutics, especially $\mathrm{T}$ and $\mathrm{B}$ cell depleting regimens, present an important risk factor for infectious complications.

Rituximab is a chimeric human-murine monoclonal antibody, designed to specifically target the transmembrane protein CD20 of B cells. The mechanism of rituximab-induced $B$ cell depletion includes antibody-dependent cell-mediated cytotoxicity (ADCC), complement-dependent cytotoxicity (CDC) and the direct induction of apoptosis, in addition to enhancing the sensitivity of $\mathrm{B}$ cells to chemotherapy and inducing cell cycle arrest. ${ }^{18}$ Rituximab can deplete peripheral B cells while B cell precursors and mature plasma cells remain relatively unaffected, allowing for a new population of B cells to develop from lymphoid stem cells. ${ }^{19}$
This remarkable activity allowed for its approval by the US Food and Drug Administration (FDA) in 1997 and European Medical Agency (EMA) in 1998 for treatment of CD20 positive cancers, including DLBCL, follicular lymphoma and chronic lymphocytic leukemia. ${ }^{19,20}$ In addition, it is also widely used in therapy of autoimmune diseases, including FDA approved therapy of rheumatoid arthritis and various off-label uses. ${ }^{21}$ In the pre-rituximab era, $\mathrm{CHOP}$ regimen was considered the best therapy for DLBCL patients. The addition of rituximab to such chemotherapy (R-CHOP) resulted in significant improvement of outcomes in DLBCL patients, therefore becoming the preferred treatment regimen for DLBCL patients. ${ }^{18}$

Rituximab shows a good safety profile with its main adverse event being infusion reactions, however concerns of increasing the risk of infections are being raised. In addition to a rapid depletion of $B$ cells, which can remain at low or undetectable levels for 2-6 months before returning to baseline levels, rituximab may also cause immunosuppression through several other mechanisms. Growing evidence, supported by an increased incidence of viral infections with the use of rituximab, now suggests rituximab also influences $\mathrm{T}$ cell immunity and predisposes patients to opportunistic infections. ${ }^{19,22}$ When administered for long periods, such as in maintenance therapy, it also causes delayed-onset neutropenia and hypogammaglobulinemia. ${ }^{22}$ Several studies have implicated B cells and antibodies (Abs) in host survival and protozoan parasite clearance, especially the B1 cell subpopulation, which appears to be evolutionary selected and maintained to facilitate prompt $\mathrm{Ab}$ responses. The B1 cells also appear to modulate $\mathrm{T}$ cell response and are implicated in the pathogenesis of toxoplasmosis through fine tune regulation of the exacerbated Th1 response by secretion of IL10, and production of Abs against heat shock protein 70 of T. Gondii. ${ }^{23}$ Parasitic infections have rarely been described in association with rituximab use ${ }^{22}$, although recently a case of reactivation of cerebral toxoplasmosis in a patient with cutaneous vasculitis was attributed to rituximab therapy. ${ }^{24}$ On the other hand, a recent study by Lanini et al. found several factors influencing the risk for infections, independent of rituximab use, among them HIV sero-status, presence of graft-versus-host-disease, type of malignancy and lymphocyte count at nadir. ${ }^{19}$

In BMT patients, the risk of latent toxoplasmosis reactivation is highest within $2-4$ months postBMT, but can be prolonged to 6 months, especially 
in cases of prolonged immune reconstitution. ${ }^{25}$ In cases of toxoplasmosis in patients with hematological malignancies, the exact time relationship between immunosuppresive therapy and reactivation of latent toxoplasma infection is not clearly established. In addition to rituximab, other immunosuppressants (e.g. fludarabine) also influence $\mathrm{T}$ cell function and this impact may persist for 1-2 years after discontinuation of therapy. ${ }^{16}$ Therefore, the emergence of febrile neutropenia in an immunosuppressed patient should be an important clinical sign, which prompts the physician to look for opportunistic infections.

Diagnosis of cerebral toxoplasmosis in immunocompromised patients is considered a medical emergency, since undiagnosed and untreated infection can rapidly be lethal. PCR testing has revolutionized the diagnosis of toxoplasmosis allowing for early detection of the parasite DNA in the CSF and blood, thereby reducing the need of direct demonstration of tachyzoites in body fluids or tissues by conventional methods, such as Giemsa staining. ${ }^{1,3}$ Serologic testing has only a limited value in immunocompromised patients, nowadays being used mostly as (i) an exclusion criterion when negative for patients, except for haematopoietic stem cell transplant patients, with symptoms consistent of acute toxoplasmosis or (ii) as a monitoring indicator, mainly for solid-organ transplant patients, prompting further investigations in cases of strong increases of IgG titers. ${ }^{1}$ An IgG avidity ratio can be helpful in establishing the diagnosis of recent toxoplasmic infection in immunocompetent patients $s^{1,3}$, with questionable results in immunocompromised patients due to their lack of ability to mount a sufficient immune response.

Treatment of cerebral toxoplasmosis comprises either (i) a combination of pyrimethamine plus sulfadiazine plus leucovorin, (ii) a combination of pyrimethamine plus clindamycin plus leucovorin or (iii) trimethoprim-sulfametoxazole. ${ }^{4,26}$ Prophylactic therapy is an important factor in toxoplasmosis prevention. In immunocompromised patients with high risk of reactivation of a latent $T$. gondii infection (eg. HIV patients with CD4+ T cell count less than 100 cells/uL or transplant patients), primary prophylaxis with trimethoprim-sulfametoxazole is usually initiated. $5,25,27$ Secondary prophylaxis guidelines for patients with HIV/AIDS who have completed initial therapy for cerebral toxoplasmosis clearly state that such patients should be administered lifelong suppressive therapy (eg. secondary prophylaxis) unless immune reconstitution occurs as a consequence of antiretroviral therapy, in which case discontinuation of treatment is indicated. ${ }^{26}$ To our knowledge, there are no such guidelines for other immunocompromised patients. Considering the fact that a combination of pyrimethamine plus sulfadiazine plus leucovorin is highly effective in HIV/AIDS patients ${ }^{26}$, we decided to proceed with secondary prophylaxis for the duration of maintenance therapy with rituximab. If rituximab was to be discontinued in the future, we planned for an additional prolongation of prophylactic therapy until rituximab-mediated depletion of B cell population would be reversed.

Within 10 days of therapy initiation, the effect of medications can already be detected with MRI; there is a decrease in the number and size of the lesions, with a reduction of edema and mass effect. Complete resolution may take as long as 6 months, and healed foci may calcify or show changes consistent with leukomalacia. ${ }^{8}$ Sometimes, a paradoxical worsening of the clinical and radiological picture is seen due to immune reconstitution inflammatory syndrome (IRIS) after initiation of therapy. ${ }^{8}$ Our patient showed a good clinical and radiological response to therapy, but further follow-up will be needed.

With this case report, we wish to emphasize the need to consider cerebral toxoplasmosis in patients with malignancies on immunosuppressive therapy when presenting with new cognitive or neurologic deficits. Although to our knowledge, no guidelines for screening of patients for latent toxoplasmosis prior to administration of potent immunosuppressants, such as rituximab, exist, we would strongly recommend it. On the basis of serology results, the need for primary prophylaxis against toxoplasmosis should then be determined for every patient.

\section{Conclusions}

Despite the widespread prevalence of latent toxoplasmosis, cerebral toxoplasmosis is an opportunistic infection that has until now only rarely been reported in patients with hematological malignancies, independent of stem cell or bone marrow transplant.

Chemotherapeutics, such as rituximab and fludarabine, probably present an important risk factor for reactivation of a latent toxoplasma infection in patients with hematological malignancies.

There are numerous differential diagnoses for cerebral toxoplasmosis, among which CNS lymphoma is the most difficult to distinguish it from. Early recognition and confirmation of cerebral 
toxoplasmosis is of essential importance, since an undiagnosed and untreated infection presents a life-threatening condition. Management of cerebral toxoplasmosis in immunocompromised patients with malignancies is currently based on guidelines for prevention and treatment of cerebral toxoplasmosis in patients with HIV/AIDS or BMT patients, for whom abundant epidemiological data exist. With the advent of new chemotherapeutic drugs there is an evolving need for recommendations for prevention and management of toxoplasmosis and other opportunistic infections in patients receiving these agents.

\section{References}

1. Robert-Gangneux F, Dardé ML. Epidemiology of and diagnostic strategies for toxoplasmosis. Clin Microbiol Rev 2012; 25: 264-96.

2. Kasper LH. Toxoplasma infections. In: Fauci AS, Kasper DL, Longo DL, Braunwald E, Hauser SL, Jameson JL, et al., editors. Harrison's principles of internal medicine. 17th Edition. New York: McGraw Hill Medical; 2008. p. 1305-11.

3. Montoya JG, Liesenfeld O. Toxoplasmosis. Lancet 2004; 12; 363(9425): 1965-76.

4. Schmidt-Hieber M, Zweigner J, Uharek L, Blau IW, Thiel E. Central nervous system infections in immunocompromised patients: update on diagnostics and therapy. Leuk Lymphoma 2009; 50: 24-36.

5. Yan J, Huang B, Liu G, Wu B, Huang S, Zheng H, et al. Meta-analysis of prevention and treatment of toxoplasmic encephalitis in HIV-infected patients. Acta Trop 2013; 127: 236-44.

6. Mele A, Paterson P, Prentice H, Leoni P, Kibbler C. Toxoplasmosis in bone marrow transplantation: a report of two cases and systematic review of the literature. Bone Marrow Transplant 2002; 29: 691-8.

7. Da Cunha S, Ferreira E, Ramos I, Martins R. Cerebral toxoplasmosis after renal transplantation. Case report and review. Acta Medica Port 1994; 7: 61-6.

8. Abdel Razek AA, Watcharakorn A, Castillo M. Parasitic diseases of the central nervous system. Neuroimaging Clin N Am 2011; 21: 815-41.

9. Offiah CE, Turnbull IW. The imaging appearances of intracranial CNS infections in adult HIV and AIDS patients. Clin Radiol 2006; 61: 393-401.

10. Masamed R, Meleis A, Lee EW, Hathout GM. Cerebral toxoplasmosis: case review and description of a new imaging sign. Clin Radiol 2009; 64: 560-3.

11. Ionita C, Wasay M, Balos L, Bakshi R. MR imaging in toxoplasmosis encephalitis after bone marrow transplantation: paucity of enhancement despite fulminant disease. Am J Neuroradiol 2004; 25: 270-3.

12. Miller RF, Hall-Craggs MA, Costa DC, Brink NS, Scaravilli F, Lucas SB, et al. Magnetic resonance imaging, thallium-201 SPET scanning, and laboratory analyses for discrimination of cerebral lymphoma and toxoplasmosis in AIDS. Sex Transm Infect 1998; 74: 258-64.

13. Camacho DL a, Smith JK, Castillo M. Differentiation of toxoplasmosis and lymphoma in AIDS patients by using apparent diffusion coefficients. Am J Neuroradiol 2003; 24: 633-7.

14. Sarrazin JL, Bonneville F, Martin-Blondel G. Brain infections. Diagn Interv Imaging 2012; 93: 473-90.

15. Maza S, Buchert R, Brenner W, Munz DL, Thiel E, Korfel A, et al. Brain and whole-body FDG-PET in diagnosis, treatment monitoring and long-term follow-up of primary CNS lymphoma. Radiol Oncol 2013; 47: 103-110.

16. Abedalthagafi M, Rushing EJ, Garvin D, Cheson B, Ozdemirli M. Asymptomatic diffuse "encephalitic" cerebral toxoplasmosis in a patient with chronic lymphocytic leukemia: case report and review of the literature. Int J Clin Exp Pathol 2009; 3: 106-9.
17. Touahri T, Pulik M, Fezoui H, Genet P, Lionnet F, Louvel D. Toxoplasmic encephalitis in a non-HIV patient with follicular lymphoma. Int J Hematol 2002; 75 : 111-2.

18. Marcus R, Hagenbeek A. The therapeutic use of rituximab in non-Hodgkin's lymphoma. Eur J Haematol Supp/ 2007; 67: 5-14.

19. Lanini S, Molloy AC, Prentice AG, Ippolito G, Kibbler CC. Infections in patients taking rituximab for hematologic malignancies: two-year cohort study. $B M C$ Infect Dis 2013; 13: 317.

20. Eisenberg R. Update on rituximab. Ann Rheum Dis 2005; 64(Suppl 4): iv55-7.

21. Gürcan HM, Keskin DB, Stern JNH, Nitzberg MA, Shekhani H, Ahmed AR. $A$ review of the current use of rituximab in autoimmune diseases. Int Immunopharmacol 2009; 9: 10-25.

22. Kelesidis T, Daikos G, Boumpas D, Tsiodras S. Does rituximab increase the incidence of infectious complications? A narrative review. Int J Infect Dis 2011; 15: e2-16.

23. Amezcua Vesely MC, Bermejo DA, Montes CL, Acosta-Rodríguez EV, Gruppi A. B-Cell response during protozoan parasite infections. J Parasitol Res 2012; 2012: 362131.

24. Safa G, Darrieux L. Cerebral toxoplasmosis after rituximab therapy. JAMA Intern Med 2013; 173: 924-6.

25. Derouin F, Pelloux H. Prevention of toxoplasmosis in transplant patients. Clin Microbiol Infect 2008; 14: 1089-101.

26. Kaplan JE, Benson C, Holmes KK, Brooks JT, Pau A, Masur H. Guidelines for prevention and treatment of opportunistic infections in HIV-infected adults and adolescents: recommendations from CDC, the National Institutes of Health, and the HIV Medicine Association of the Infectious Diseases Society of America. MMWR Recomm Rep 2009; 58(RR-4): 1-207.

27. Soave R. Prophylaxis strategies for solid-organ transplantation. Clin Infect Dis 2001; 33(Suppl 1): S26-31. 